\begin{abstract}
The future generation of modern illumination should not only be cheap and highly efficient, but also demonstrate high quality of light, light which allows better color differentiation and fidelity. Here we are presenting a novel approach to create a white solid-state light source providing ultimate color rendition necessary for a number of applications. The proposed semi-hybrid device combines a monolithic blue-cyan light emitting diode (MBC LED) with a green-red phosphor mixture. It has shown a superior color rendering index (CRI), 98.6, at correlated color temperature of around $3400 \mathrm{~K}$. The MBC LED epi-structure did not suffer from the efficiency reduction typical for monolithic multi-color emitters and was implemented in the two most popular chip designs: "epi-up "and "flip-chip ". Redistribution of the blue and cyan band amplitudes in the white-light emission spectrum, using the operating current, is found to be an effective tool for fine tuning the color characteristics.
\end{abstract}
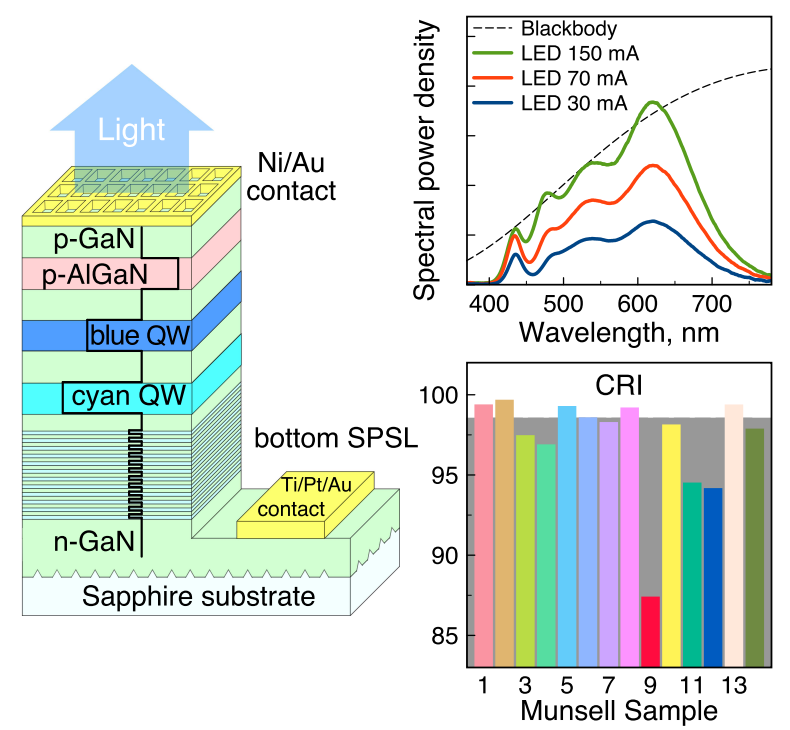

\title{
Superior color rendering with a phosphor-converted blue-cyan monolithic light-emitting diode
}

\author{
llya E. Titkov ${ }^{1}$, Amit Yadav ${ }^{1, *}$, Sergey Yu. Karpov ${ }^{2}$, Alexei V. Sakharov ${ }^{3}$, An- \\ drey F. Tsatsulnikov ${ }^{3,4}$, Thomas J. Slight ${ }^{5}$, Andrei Gorodetsky ${ }^{1}$ and Edik U. Rafailov ${ }^{1}$
}

\section{Introduction}

Development of light emitting diode (LED)-based solid state lighting has led to an understanding that various lighting applications provide very different requirements to the quality and characteristics of white light. The mainstream applications, like outdoor (street) and indoor lightings are primarily aimed at saving electricity consumption and, therefore, need maximum efficacy and minimum cost of white-light sources at rather moderate demands for color rendition: CRI of about $80-85$ is considered as quite acceptable in this case $[1,2]$. Nevertheless, there are many important applications that require superior color rendition.

True rendition of colors can significantly improve the appearance of objects, particularly in super-markets, museums, galleries, and exhibitions where visual presentation is critical. Such kind of illumination is also very important in specific areas such as hospitals, eye centers, drawing tools, photo/video recording or textile industries where color differentiation is vital. For example, Figure 1 represents the set of Munsell targets illuminated with two different light sources with low and high CRIs. The right hand picture clearly demonstrates better color differentiation.

Žukauskas et. al. [3] have suggested theoretical spectral optimization for white phosphor-converted LEDs (pc-LEDs) to achieve high CRI 96-98. These light sources combine the light produced by a blue LED with broad-band emission of two or three phosphors, providing luminous efficacy of radiation (LER) in the range of 234-285 $\mathrm{lm} / \mathrm{W}$, which is close to LER 260-280 $\mathrm{lm} / \mathrm{W}$ estimated for the sun radiation spectrum truncated at the level of $1 \%$ of the maximum sensitivity of human eye [4].

The above approach corresponds to a practical one based on the mixture of two or more phosphors to achieve quasiuniform emission across the visible spectrum, $\lambda=400-730$ $\mathrm{nm}$. The major manufacturers, like Nichia, CREE, Osram, Yuji International, etc. can produce high-CRI (up to 9598 [5-7]) LEDs covered by the phosphor mixtures based on Yttrium Aluminum Garnet (YAG) (green/yellow: 540-

\footnotetext{
${ }^{1}$ Aston Institute of Photonic Technologies, Aston University, B4 7ET, Birmingham, UK

2 STR Group Soft-Impact, Ltd., P.O.Box 83, 27 Engels ave., 194156 St.Petersburg, Russia

${ }^{3}$ Ioffe Institute, 26 Polytekhnicheskaya str., 194021 St.Petersburg, Russia

4 ITMO University, St. Petersburg, 197101, Russia

${ }^{5}$ Compound Semiconductor Technologies Global Ltd, 4 Stanley Boulevard, Hamilton International Technology Park, Hamilton, G72 0BN, Scotland, UK
} 


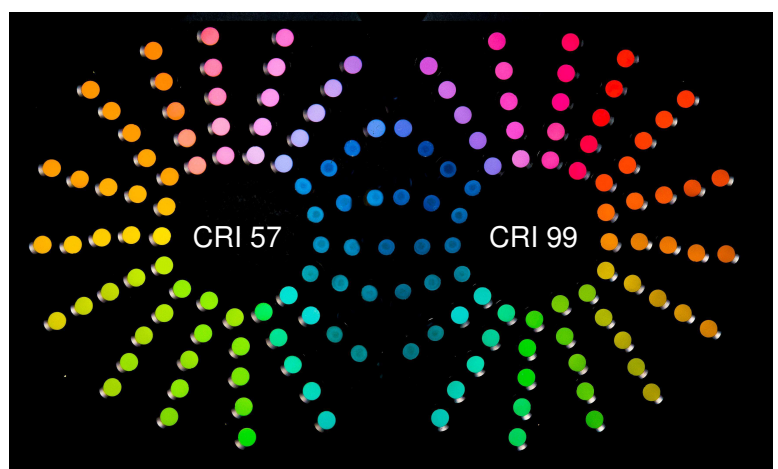

Figure 1 Color Munsell targets illuminated by an office luminescence lamp with CRI 57 (left), and halogen lamp with CRI 99 (right).

$560 \mathrm{~nm}$ ), Lutetium Aluminum Garnet (green: 520-540 nm), Nitrides (red: 615-660 nm) and Oxynitrides (greenish-red: $500-650 \mathrm{~nm}$ ), providing full visible spectrum coverage [8]. At the same time some researchers create new phosphor materials to improve color rendition [9-11].

The highest known CRI value, 99.1, has been demonstrated 7 years ago [12] by using three remote phosphors excited by a violet LED (405 nm). The efficacy of the pc-LED was $59 \mathrm{~lm} / \mathrm{W}$, which is quite acceptable for such kind of device. Nevertheless, there is a lack of competitive products with perfect color rendition on the market, since handling time and production cost is affected with increasing number of phosphors. To address this issue, research aimed at achieving ultimate color rendition with minimal production expense is still required [1].

While the use of many phosphors for light conversion is rather reliable, it is not always the optimal solution. First, practically all the commercially available phosphors possess broad excitation and emission spectral bands. So, their overlap, especially pronounced in mixtures of phosphors disturbs the additivity of their emission spectra, making their control difficult. Second, the light conversion efficiency of every phosphor approaches its maximum value at a certain size of the phosphor particles. This size also influences dramatically the pattern of light scattering by the particles, which determines eventually the angular uniformity of color characteristics. Therefore, simultaneous optimization of the color uniformity and conversion efficiency of the phosphor mixture becomes more challenging with increasing the number of phosphors. Third, the use of every additional phosphor introduces additional losses originated from the Stokes shift of the photon energy by light down-conversion. Therefore, there exists a practical trend of reducing the number of phosphors employed for white light production. This trend lies in the mainstream of future research and developments on lighting technologies. In particular, smart sources with controllable variation of the white-light parameters, e. g. of correlated color temperature (CCT), are much easy achievable by using LEDs without phosphor [13] than by pc-LEDs. Another direction, namely Li-Fi communication, [14] re- quires fast switching of the LED emission, including white light. Since, the luminescence lifetime of various phosphors differ considerably from each other, i. e. from $\sim 60 \mathrm{~ns}$ for YAG: $\mathrm{Ce}^{3+}$ yellow phosphor [15] to $\sim 600-800 \mu$ s for red $\mathrm{Y}_{2} \mathrm{Mo}_{4} \mathrm{O}_{15}: \mathrm{Eu}^{3+}$ one, [16] reducing the number of phosphors in a white-light source facilitates selection of those possessing the most fast emission kinetics.

An alternative approach to white-light generation is based on mixing the light emitted by various LEDs without any phosphors. Theoretical optimization of such a light source [13] has shown that (i) its efficacy is systematically lower than that of pc-LEDs and (ii) the CRI obtained is limited by the value of $\sim 90-92$, which is unacceptable for ultimate color rendition. The first limitation arises from the well-known "green gap" problem [17] the nature of which is still under investigation [18], whereas the second one is caused by relatively narrow emission spectra of individual LEDs [3]. Though CRI can be improved by using four or more individual LEDs, the increase of the number of LEDs is predicted to be accompanied by a dramatic losses in the efficacy of such a white-light source because of the "green gap" [13]. This shows that utilization of phosphors is still unavoidable in the state-of-the-art LED fabrication technology.

A compromise between the above alternative approaches is provided by the Brilliant Mix concept [19] utilizing partial conversion of blue III-N LED light by a green phosphor combined with the red light emission from red AlInGaP LED. Minimizing the number of phosphors employed, the concept has proved remarkable improvement in the color rendition $(C R I \sim 92)$ at a rather high efficacy (more than 100 $\mathrm{lm} / \mathrm{W})$. The cost to be paid for this is the necessity of complex current driving of the LEDs made of different material systems and control of their thermal stability. In addition, the demonstrated CRI value requires further improvement for superior color rendering.

Additional opportunities can be discovered by the use of multi-color LEDs providing emission of photons in different spectral bands from the same devices. In particular, generation of white light by such LEDs is also possible [20]. The multi-color LEDs utilize normally monolithic integration of various active regions in planar [21,22] or non-planar [23,24] structures. Efficiency of such devices is still low, depending on combination of the emission wavelengths [25], but it is sufficient for a number of specific applications like, for instance, quantum photonic imagers [26]. Due to the "green gap" problem, it is difficult to get efficient white emitters based on RGB mixing in multi-color monolithic structures but the use of additional emission bands seems to be helpful for improving color rendition in $\mathrm{PC}$ LEDs.

Recently, the use of dual-wavelength PC-LEDs with a yellow YAG:Ce ${ }^{3+}$ phosphor has been suggested by simulations for improving the color rendition of the commonly exploited color mixing scheme [27]. Assuming a Gaussian shape of both LED emission lines with the same width and using incomplete optimization of the color mixing (the magnitude ratio of two emission lines was fixed), improvement in CRI from $78\left(\mathrm{CCT}=4000 \mathrm{~K}\right.$ at $\left.\lambda_{\mathrm{p}}=475 \mathrm{~nm}\right)$ for the 
(a)
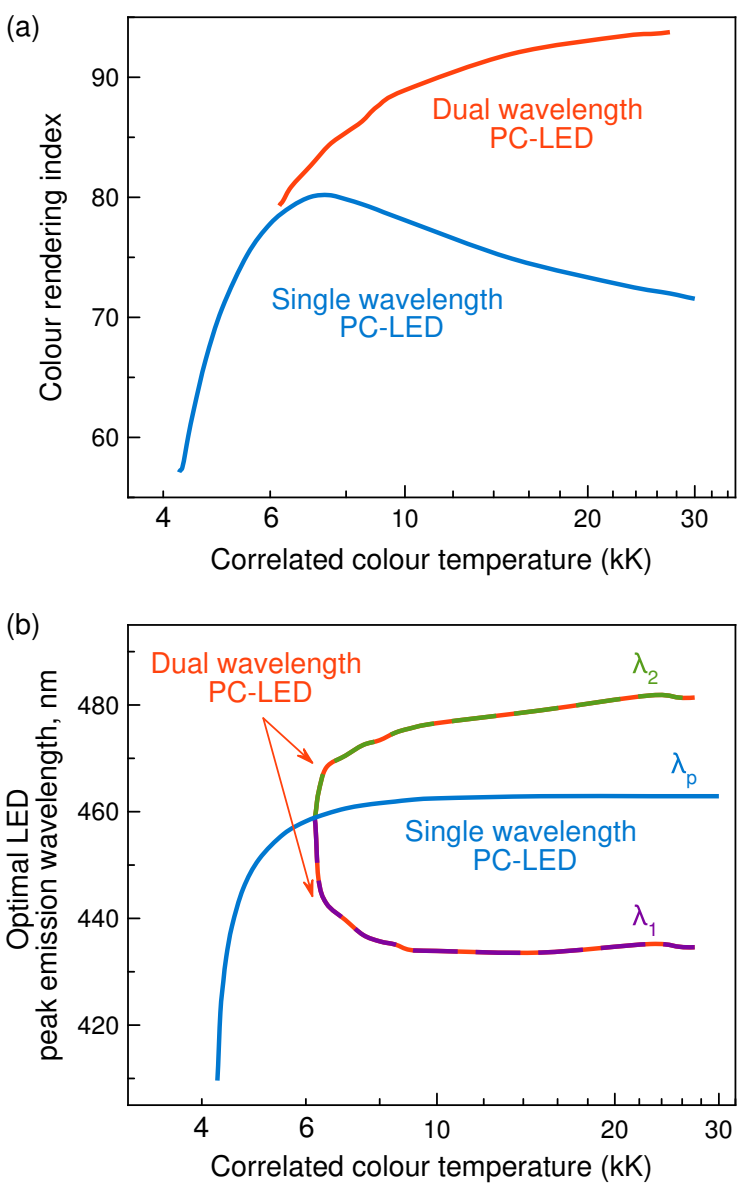

Figure 2 General CRI of the white light metameric with blackbody radiation as a function of CCT obtained at optimized color mixing from a single-wavelength/dual-wavelength LED and a YAG:Ce ${ }^{3+}$ yellow phosphor (a). Optimal peak emission wavelengths for single-wavelength $\left(\lambda_{p}\right)$ and dual-wavelength $\left(\lambda_{l}\right.$ and $\lambda_{2}$ ) LEDs (b).

reference blue pc-LED to $91\left(\mathrm{CCT}=5800 \mathrm{~K}\right.$ at $\lambda_{1}=435$ $\mathrm{nm}$ and $\lambda_{2}=490 \mathrm{~nm}$ ) has been predicted.

Our simulations with the realistic asymmetric emission spectra and wavelength-dependent widths [13] provide rather different results presented in Figure 2. Firstly, a singlewavelength pc-LED $\left(\lambda_{\mathrm{p}}<460 \mathrm{~nm}\right.$ and YAG:Ce ${ }^{3+}$ phosphor $)$ is predicted to provide a higher CRI (up to 80) at CCT $<6000 \mathrm{~K}$ than dual-wavelength PC-LEDs. Secondly, CRI greater than 80 can be attained by using the dual-wavelength pc-LEDs (Fig.2a). However, this is predicted to occur only at high CCTs that are off practical importance. Besides, the maximum achievable CRI values of less than 94 are yet too low for ultimate color rendition, first of all, because of lacking red component in the total emission spectrum.

The idea of using dual-wavelength monolithic LEDs for CRI improvement was tested experimentally by Osram OS and an increase in general CRI $\left(R_{a}\right)$ from 67 to 76 at $\mathrm{CCT}=6500 \mathrm{~K}$ was observed without sacrificing emission efficiency [28]. The level of the improvement correlates well with our simulations shown in Fig.2. Unfortunately, the use of only yellow phosphor for light conversion did not allow achieving of excellent high color rendition [27] as well as the use of only blue excitation with yellow and red phosphors [29] (also see Sec. 3.2).

In this paper, we show experimentally that superior color rendition can be, nevertheless, achieved from monolithic dual-wavelength pc-LEDs by using a mixture of only two phosphors emitting in the green and red spectral ranges.

\section{Materials and Methods}

Monolithic blue-cyan (MBC) epi-structure (Fig.3a) was grown by AIX2000HT MOCVD reactor on a C-plane sapphire substrate patterned with micro-cones [30] for better light extraction. The LED active region consisted of a $3 \mathrm{~nm}$ InGaN QW, emitting in the cyan (460-475 nm) spectral range ("cyan " $Q W$ ) and a $3 \mathrm{~nm}$ InGaN quantum well (QW), emitting in the blue (430-435 nm) spectral range ("blue "QW), both grown on top of 12-period InGaN/GaN short-period superlattice [31]. The wafers were processed by CSTG Ltd. as epi-up LED chips with semitransparent golden-grid contact electrodes on the top of the mesa (Fig.3b). The mesa size was $240 \times 320 \mu \mathrm{m}^{2}$; the chips were mounted onto aluminium star-like plates for better heat sinking. For the partial blue/cyan light conversion we used a commercial 1-mm thick phosphor plastic panel (RF3000K-96) designed by Phosphortech Corporation. The panel contained a mixture of red and green phosphors based on the patented YAG and Nitride composition [32]. The phosphor is optimized to provide white light with CRI $=96$ at $\mathrm{CCT}=3000 \mathrm{~K}$ by pumping with a blue $(450 \mathrm{~nm}) \mathrm{LED}$. In our experiments, a cylindrical geometry of the remote phosphor cover (Fig.3c) was employed for its simplicity and symmetry, considering wide angular emission from the surface emitting devices. The cylinder used was $20 \mathrm{~mm}$ in height and $18 \mathrm{~mm}$ in diameter. The LED chip was placed in the bottom center of cylinder. The yellow color at the top of the cover was deeper than in the bottom, as it is seen in Fig. $3 c$, depending also on the observation angle. This is related to the enhanced scattering of photons emitted by LED from both horizontal and vertical surfaces of the converter. In practice, the observed non-uniformity may be avoided by optimization of the cover shape.

The optical parameters of the devices were measured in CW regime with a compact spectrometer CDS-600 connected with 10-inch integration sphere from Labsphere Co, Ltd. and Keithley-2400 programmable current source. The spectral data processing was done with Pro-Lite LightMtrx software using the CIE 1931 metrics [33].

\section{Results and discussion}

Experimental results are presented in the next two subsections. One describes characteristics of the bare MBC LED chip (Fig.3b) necessary for further discussion, whereas another one considers operation of the LED covered with remote phosphor converter (Fig.3c). 


\subsection{Characterization of bare $M C B-L E D$}

First, an MBC LED has been characterized by measuring its external quantum efficiency (EQE) as a function of operating current. In order to estimate IQE of the LED and the efficiency of light extraction from the LED die, we have applied an original method recently suggested [34]. The method operates with normalized optical power $p$ which is the ratio of the LED output power at a certain current to its value $P_{m}$ corresponding to the maximum of external quantum efficiency $\mathrm{EQE}_{\max }$. Plotting the $\mathrm{y}(\mathrm{x})=\mathrm{EQE}_{\max } / \mathrm{EQE}$ ratio versus $\mathrm{X}=\left(p^{1 / 2}+p^{-1 / 2}\right)$ and approximating the experimental points by a straight line (see Fig.4b) let us to determine the quality factor $Q$ of the LED structure, maximum IQE value $\mathrm{IQE}_{\max }=Q /(Q+2)$, and light extraction efficiency (LEE) $\eta_{\text {ext }}=\mathrm{EQE}_{\max } / \mathrm{IQE}_{\max }$. As a result, $Q=$ $3.4, \mathrm{IQE}_{\max }=63 \%$, and $\eta_{\text {ext }}=14.8 \%$ have been derived from the characterization data.

The measured $\operatorname{EQE}(p)$ function is then well approximated by the theoretical curve $\mathrm{EQE}=\eta_{\text {ext }} Q /\left(Q+p^{1 / 2}+p\right.$ $-1 / 2)$, except for high $p$ values greater than $\sim 15-20(\mathrm{x}>4.5)$,

(a)

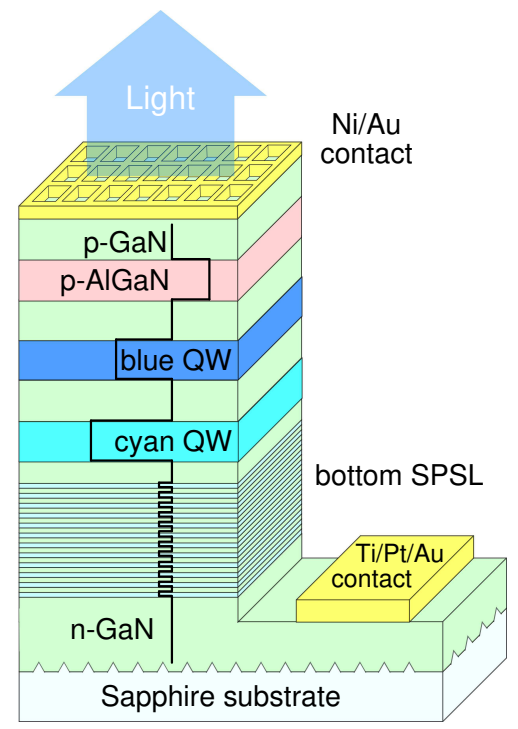

(b)

(c)
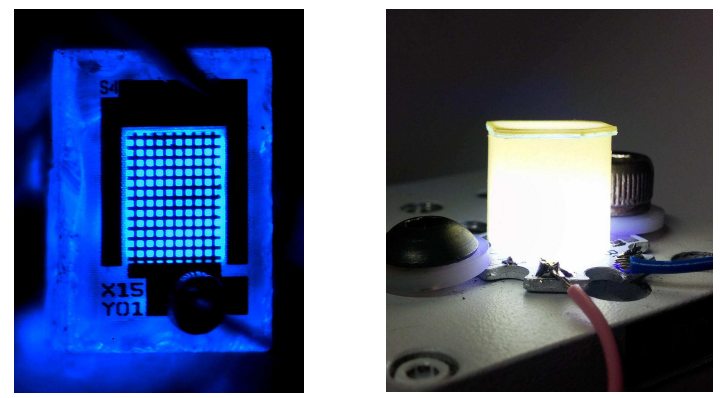

Figure 3 Schematic sandwich structure and band gap profile (black solid line) in MBC LED structure (a), micrograph of the operating MBC LED chip (b), and photograph of the remote phosphor converter of a cylindrical shape (c). see Fig.4. The discrepancy between the characterization data and theoretical predictions is caused by dramatic increase in the contribution of the cyan QW to the photon emission. Indeed, at the currents less than $\sim 30 \mathrm{~mA}(\mathrm{p}<10)$ a blue shift of the centroid emission wavelength is observed along with the practically constant spectrum band width (FWHM). As it follows from Fig.5a comparing the MBC LED emission spectra at various operating currents, the emission from the blue QW dominates under these conditions. So, the behavior of the emission wavelength and FWHM corresponds here to that commonly observed in nitride LEDs where the blue shift is conventionally interpreted in terms of screening the polarization field in the InGaN QW. At the currents greater than $\sim 60-80 \mathrm{~mA}(\mathrm{p}>15-20)$ the blue shift is changed into a strong red shift with simultaneous increase in FWHM. According to Fig.5a, this is accompanied by a rise in the cyan band (460-475 nm) intensity, so the centroid wavelength becomes red-shifted and the spectrum width becomes enlarged due to merging blue and cyan peaks. Since the cyan band has a lower efficiency, being closer to the "green gap", its growing contribution at high currents makes EQE lower than it is predicted by theoretical approximation.

In contrast to the centroid wavelength, the peak wavelengths for blue and cyan emission individually do not exhibit a noticeable red shift with current (see Fig.5a). That means negligible impact of the self-heating on the total emission spectrum of the MBC LED. The interplay between contributions of the blue and cyan QWs to the total spectrum is strongly current-dependent which enables us to fine tune the color characteristics of pc-LED by adjustment of the current.

\subsection{Characterization of $p c-L E D$}

Emission spectra on white-light pc-LEDs measured at different operating currents are shown in Fig.5b. Comparison of the spectra with those plotted in Fig.5a demonstrates substantial redistribution in the magnitudes of blue and cyan emission peaks, caused by stronger absorption of blue light by the phosphor mixture, as compared to cyan light. This absorption selectivity is quite beneficial for improvement of color rendition of the pc-LED. Indeed, its emission spectrum is almost filling the whole visible spectral range in the manner like the black-body radiation at the corresponding temperature (see dash line in Fig.5b). At that, the magnitude of the cyan peak should be higher than the magnitude of the blue peak to provide ultimate color rendition. Such a ratio of the peak magnitudes is not available in the bare MBC LED (Fig.5a) but it becomes feasible in pc-LED due to the spectral selectivity of light absorption by phosphors (Fig.5b). One can also see from Fig.5b that increase in the operating current results in ever growing cyan emission band in the pc-LED emission spectrum. Further, the chromaticity coordinates are fairly stable in the current range of $30 \mathrm{~mA}-150$ $\mathrm{mA}$ as one can see from Fig.5c.

Figure 6 presents the evolution of the main color characteristics of the pc-LED with current. First, CCT is found to be rather stable with current, decreasing from $\sim 3500 \mathrm{~K}$ at 1 
(a)
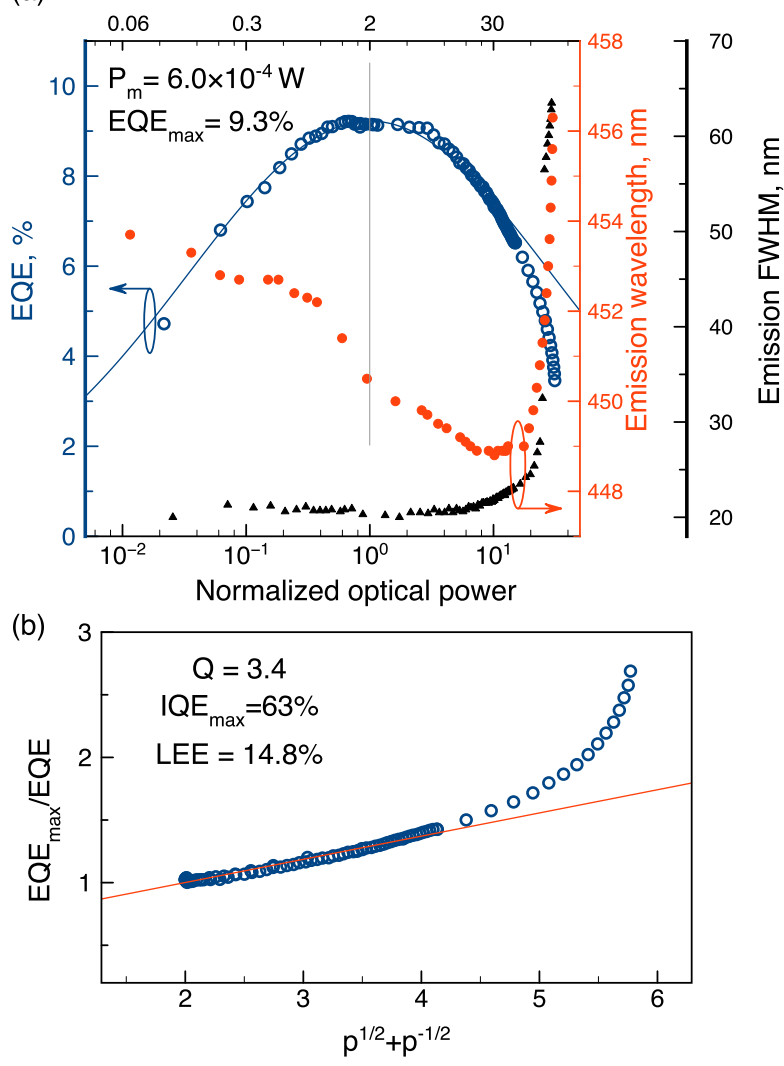

Figure 4 (a) EQE, centroid emission wavelength (red circles), and emission spectrum FWHM (black triangles) of bare dualwavelength LED as a function of normalized optical power $(p)$ (bottom scale), and drive current (top scale); blue solid line shows EQE theoretical fitting curve (b) fitting (red line) of the characterization data (blue circles) by ABC-model to determine LEE and IQE.

$\mathrm{mA}$ to $\sim 3300 \mathrm{~K}$ at $200 \mathrm{~mA}$ (Fig.6a). Second, luminous efficacy of radiation (LER) varies between 262 and $282 \mathrm{~lm} / \mathrm{W}$, approaching its maximum at $10 \mathrm{~mA}$. The above LER values agree well with those reported for truncated sun-radiation spectrum [4].

To characterize color rendition, we have determined general color rendering indices (CRIs) by using eight, $R_{a}(8)$, and fourteen, $R_{a}(14)$, standard Munsell samples. At low currents, where the blue emission band dominates, $R_{a}(8)$ is found to correspond to the nominal value of 96 reported by the phosphor vendor. Increase in the operating current results in growth of the cyan emission band and, hence, in $R_{a}(8)$ increase up to 98.6 at $80 \mathrm{~mA}$ (Fig.6a). Further rise of the operating current leads to a dramatic reduction of $R_{a}(8)$, which correlates with the onset of strong red shift and broadening of the bare MBC LED emission spectrum (see Fig.4a). Variation of $R_{a}(14)$ with current is qualitatively similar to that of $R_{a}(8)$ (Fig.6a). In order to check, if it is possible to obtain such a high CRI by using a singlewavelength LED excitation, we have replaced the MBC LED with a blue one $(\sim 455 \mathrm{~nm})$ in the same experimental (a)

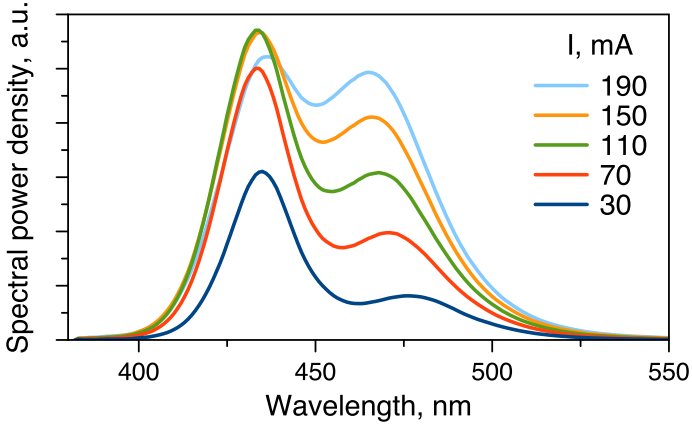

(b)

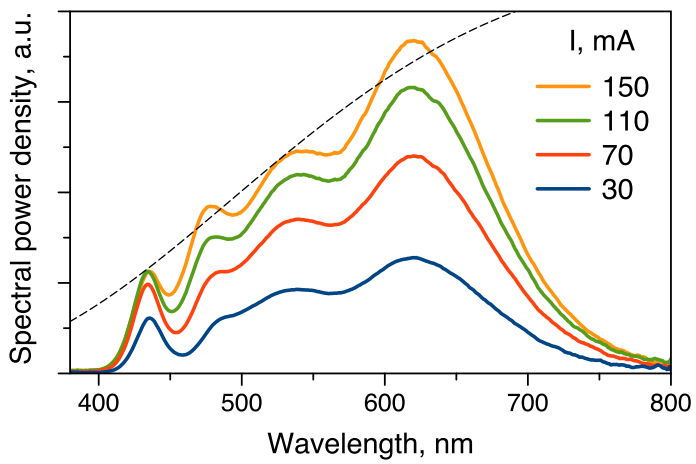

(c)

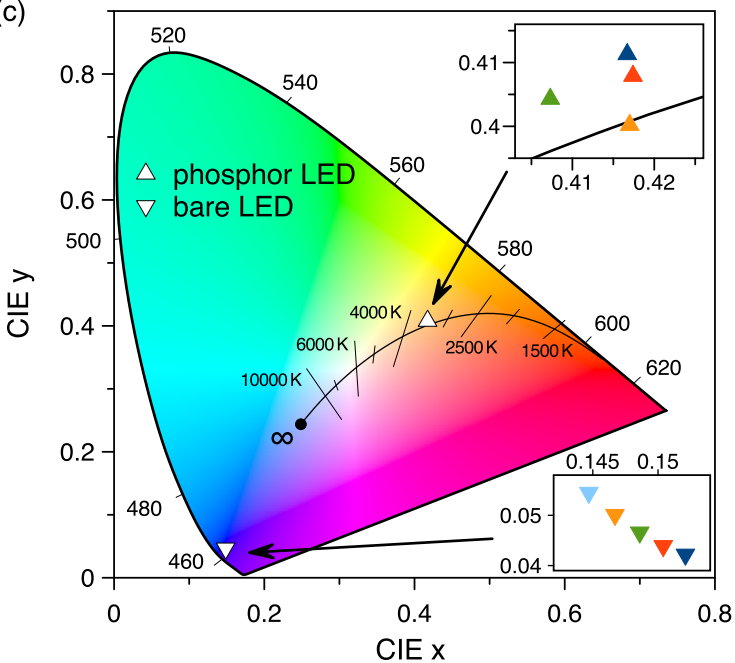

Figure 5 (a) Emission spectra of bare blue/cyan monolithic chip; (b) white-light pc-LED measured at different operating currents; dash line represents the black-body radiation spectrum at $3400 \mathrm{~K}$ and (c) CIE 1931 diagram depicting the chromaticity coordinates corresponding to the EL spectra in (a) and (b); Top inset - planckian locus (black line) and chromaticity coordinates corresponding to pc-LED; Bottom inset - coordinates corresponding to blue/cyan monolithic LED.

setup. The measurements of the color characteristics of such a reference pc-LED have shown $\operatorname{Ra}(8)$ to increase from $\sim$ 84 at low currents to 96.0 and then to decrease gradually to 95.0 at high currents. This result demonstrates clearly the important role of the cyan spectral band in the spectra of MBC LED for further improving the color rendition. 

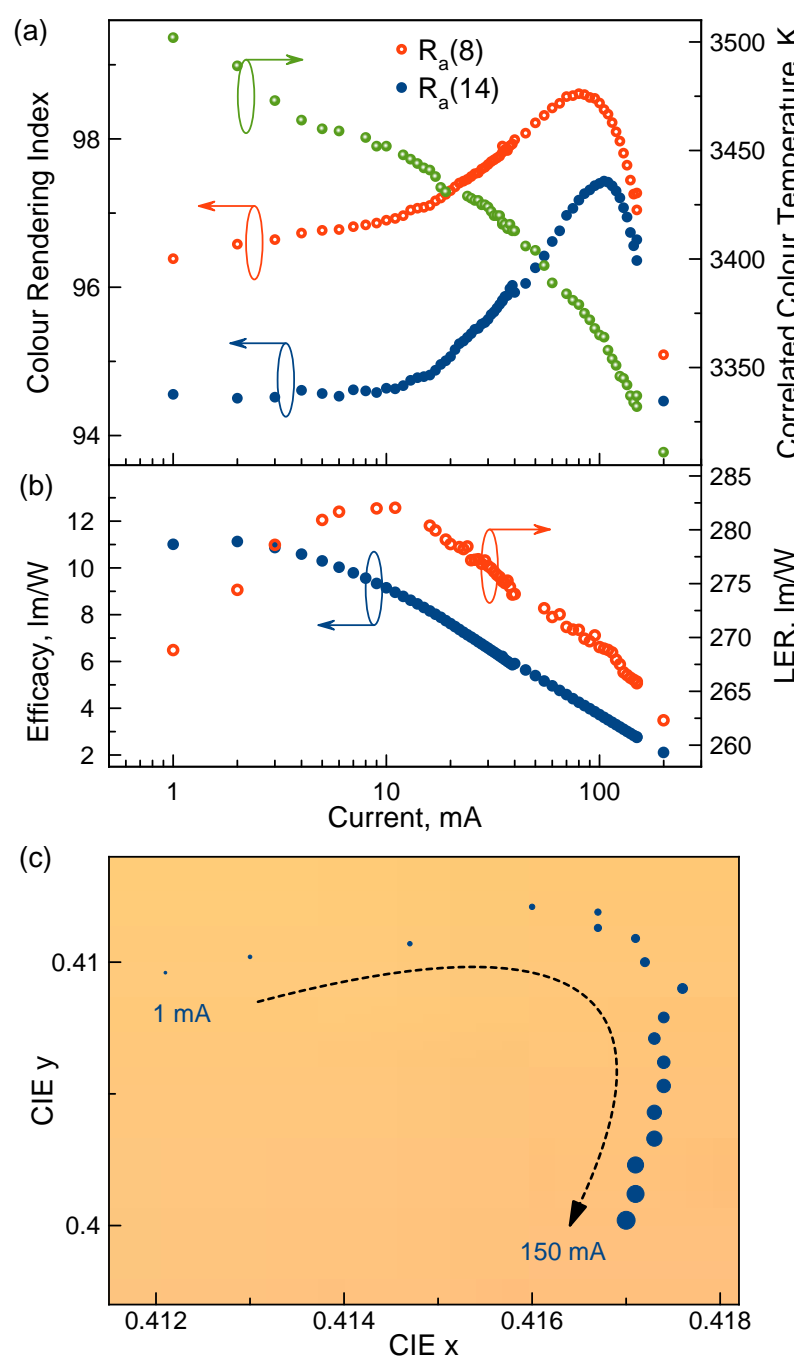

Figure 6 Color characteristics of blue/cyan pc-LEDs as a function of current. General CRI and CCT determined for $8\left[R_{a}(8)\right]$ and 14 $\left[R_{a}(14)\right]$ standard Munsell samples (a); Luminous Efficacy and Luminous Efficacy of Radiation (b); (c) Evolution of the chromaticity coordinates $x$ and $y$ vs. injection current (solid circles) where the circle size shows increasing current and radiant flux.

So, tuning the pc-LED operating currents enables achieving ultimate color rendition with $R_{a}(8)=98.6$ and $R_{a}(14)$ $=97.4$. Comparison of the partial CRIs obtained for low (Fig.7a) and optimal (Fig.7c) current shows that all the 14 partial CRIs become greater than 94 at optimal pumping, except for $R_{9}$ which is still less than 88 . One can see that $R_{a}(8)$ and $R_{a}(14)$ improvements are related to increasing of all partial CRIs and, especially, of $R_{3}, R_{4}, R_{11}$ and $R_{12}$. The partial CRIs (except $R_{8}$ and $R_{14}$ ) drop significantly at high currents $>150 \mathrm{~mA}$ (see Fig.7b) because of rapidly increasing cyan emission power. In spite of $R_{9}$ growing at high current the decrease of $R_{1}$ and $R_{5}-R_{7}$ leads to decrease of general index $\left(R_{a}\right)$ down to 97 at $150 \mathrm{~mA}$ and then to 95.6 at $190 \mathrm{~mA}$. Still a relatively low $R_{9}$ is, in particular, the major reason for the difference between $R_{a}(8)$ and $R_{a}(14)$.
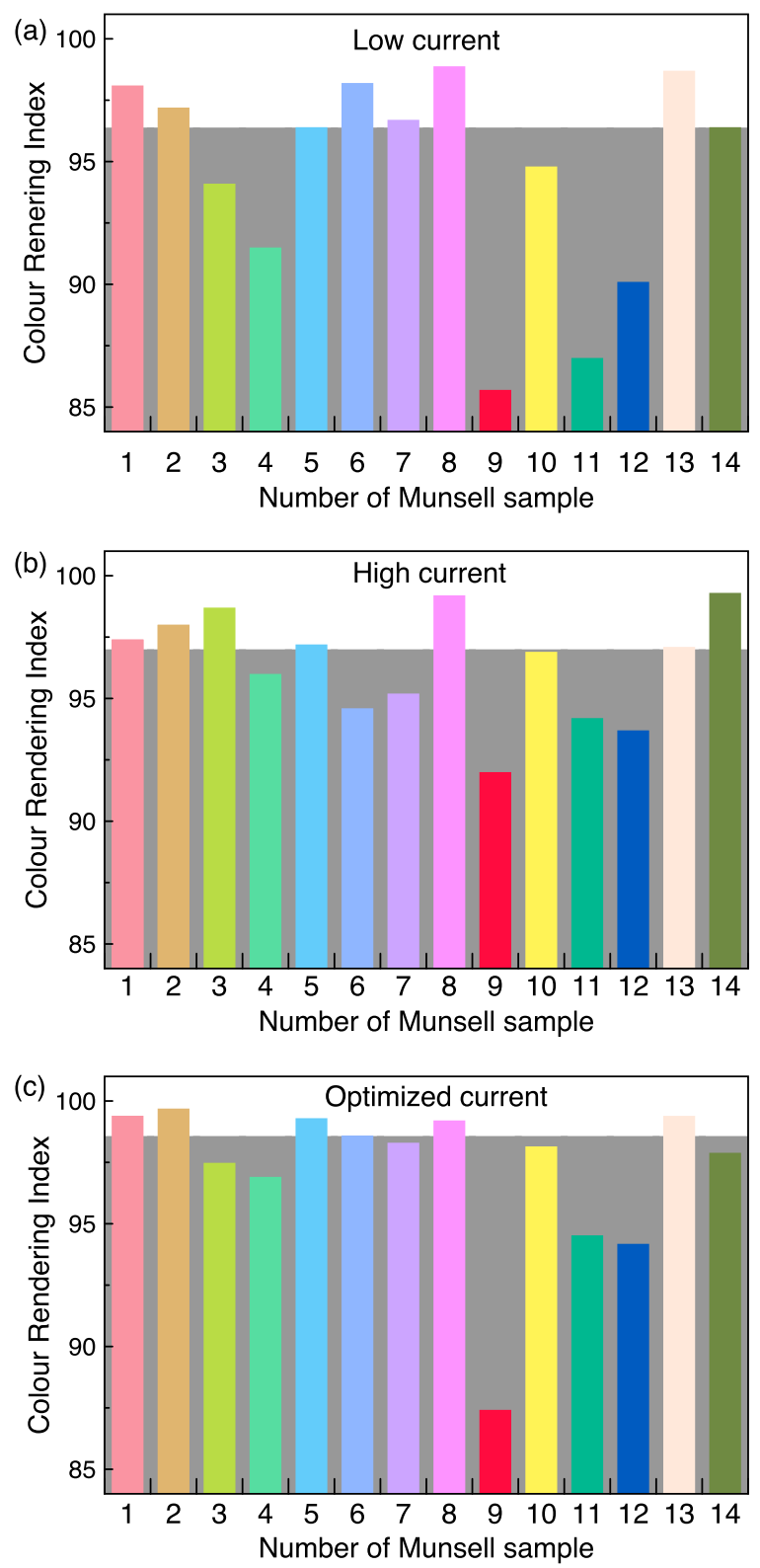

Figure 7 Partial color rendering indices for the main Munsell samples for (a) low (1 mA), (b) high (150 mA) and (c) optimal (80 $m A)$ LED operation currents. The background shadow in each plot indicates the averaged color rendering index $R_{a}(8)$.

The above results show also that the relatively low $R_{9}$ remains yet the bottleneck for the ultimate color rendition of the studied white-light LED. The reason for that is the decay of the red phosphor emission intensity at the wavelength longer than $\sim 650 \mathrm{~nm}$ (see Fig. $5 \mathrm{~b}$ ), which slightly lowers $R_{l 1}$ and $R_{12}$ as well. A natural remedy for the $R_{9}$ improvement would be the use of another red phosphor with wider emission band. This is expected, however, to result in the efficacy losses caused by extension of the red phosphor emission spectrum beyond the spectral range of the human eye receptivity. In contrary, an alternative approach, which seems 
to be promising, implies utilization of a narrow-band red phosphor [10] with the peak emission wavelength shifted towards $700 \mathrm{~nm}$. Now it is still unclear whether the use of such a phosphor may improve $R_{9}$ without remarkable losses in the efficacy and other partial color rendering indexes or not. So, the trade-off between the $R_{9}$ value and efficacy of the white-light source or other partial CRIs seems us to be the issue for the further studies.

\subsection{Assessment of practical performance}

In the case of pc-LED, the epi-up chip design (Fig.3a) results in the maximum luminous efficacy $11 \mathrm{~lm} / \mathrm{W}$ and $4 \mathrm{~lm} / \mathrm{W}$ at the operation current providing maximum CRI (Fig.6b). The low LED efficacy is primarily caused by the non-optimal chip design with $\mathrm{LEE}=14.8 \%$ only.

An alternative MK-24 $1310 \times 1310 \mu \mathrm{m}^{2}$ flip-chip design [35] providing $\mathrm{LEE}=43 \%$ has demonstrated the maximum efficacy $33 \mathrm{~lm} / \mathrm{W}$ and efficacy $24 \mathrm{~lm} / \mathrm{W}$ at optimized current without reducing the maximum $R_{a}(8)$ value. Using state-ofthe-art chip design developed for high-power LEDs, LEE can be additionally improved, at least, up to $\sim 82-83 \%$ in the spectral range of the dual-wavelength LED operation [36]. Moulding the chip package with silicone lens can give further improvement of LEE, up to 90\% [37].

Extrapolating experimentally obtained LEE of these two LED chips we can estimate possible efficacy of the dual-wavelength pc-LED $\sim 50-70 \mathrm{~lm} / \mathrm{W}$, which is already acceptable for practical applications. Further increase of the efficacy would require improvements of the IQE of the dual-wavelength active region.

Another room for the efficacy improvement gives tuning of the blue/cyan ratio in the emission spectrum in a way to maximize CRI at the operation current corresponding to the maximum EQE. This can be done by adjusting electrical properties of the spacer separating blue and cyan QW in the LED active region [25].

In order to improve further $R_{9}$ and, thus, general CRIs, fine adjustment of the red phosphor peak wavelength and its spectrum width is needed. This work is planned to be done in the future studies.

\section{Conclusion}

It has been demonstrated for the first time that combination of a blue/cyan dual-wavelength monolithic LED emission with two-phosphor mixture enables development of the warm white-light source $(\mathrm{CCT} \sim 3400 \mathrm{~K})$ with superior color rendition: $R_{a}(8)=98.6$ and $R_{a}(14)=97.4$. The feasible improvements in the LED structure and chip design enable approaching the efficacy sufficient for practical applications. Further CRI increase is expected from the optimization of the white-light spectrum aimed at focused improvement of the $R_{9}$ partial CRI. Two alternative approaches suitable for this are discussed in the paper. Both of them require a careful optimization of the red phosphor characteristics in the future studies.
The suggested solution has two main advantages: first, it operates with a minimum number of phosphors capable of providing high CRI values without interfering with each other because of substantial overlapping of the their excitation and emission curves. Second, the monolithic LED contains two active regions emitting light beyond the "green gap" where the emission efficiency drops dramatically. The latter allows one to get rather high IQEs of both QWs, which is necessary for the overall pc-LED performance. To increase the efficacy of the pc-LED, both the use of advanced chip designs and further increase of the dual-wavelength LED efficiency are necessary.

Acknowledgements. This work was supported by European Union FP7, NEWLED project, Grant number 318388.

Authors would also like to thank Magicplot LLC for providing Magic Plot Pro plotting and fitting software used for preparation of all plots and figures in this manuscript.

Key words: blue-cyan monolithic LED, novel semi-hybrid approach, superior $\mathrm{CRI}$, two phosphor mix

\section{References}

[1] J. Y. Tsao, M. H. Crawford, M. E. Coltrin, A. J. Fischer, D. D. Koleske, G. S. Subramania, G. T. Wang, J. J. Wierer, and R. F. Karlicek, Adv. Opt. Mater. 2(9), 809-836 (2014).

[2] M. Crawford, IEEE J. Sel. Top. Quantum Electron. 15(4), 1028-1040 (2009).

[3] A. Žukauskas, R. Vaicekauskas, F. Ivanauskas, H. Vaitkevičius, and M.S. Shur, Appl. Phys. Lett. 93(5) (2008).

[4] T. W. Murphy, J. Appl. Phys. 111(10), 104909 (2012).

[5] Y. Narukawa, J. Narita, T. Sakamoto, T. Yamada, H. Narimatsu, M. Sano, and T. Mukai, Phys. status solidi 204(6), 2087-2093 (2007).

[6] Yuji International Co. Ltd., High CRI LED for lighting, 2016, http://www.yujiintl.com/high-cri-led-lighting.

[7] OSRAM Opto Semiconductors, High CRI LEDlights, 2016, https://ledlight.osram-os.com/2011/04/ high-color-rendering-index-cri-led-lights/.

[8] N. Kimura, K. Sakuma, S. Hirafune, K. Asano, N. Hirosaki, and R. J. Xie, Appl. Phys. Lett. 90(5), 051109 (2007).

[9] X. Li, J. D. Budai, F. Liu, J. Y. Howe, J. Zhang, X. J. Wang, Z. Gu, C. Sun, R. S. Meltzer, and Z. Pan, Light Sci. Appl. 2(1), e50 (2013).

[10] D. Chen, Y. Zhou, W. Xu, J. Zhong, Z. Ji, and W. Xiang, J. Mater. Chem. C 4(8), 1704-1712 (2016).

[11] D. Chen, W. Xiang, X. Liang, J. Zhong, H. Yu, M. Ding, H. Lu, and Z. Ji, J. Eur. Ceram. Soc. 35(3), 859-869 (2015).

[12] T. Fukui, K. Kamon, J. Takeshita, H. Hayashi, T. Miyachi, Y. Uchida, S. Kurai, and T. Taguchi, Jpn. J. Appl. Phys. 48(11), 112101 (2009).

[13] K. A. Bulashevich, A. V. Kulik, and S. Y. Karpov, Phys. status solidi 212(5), 914-919 (2015).

[14] S. Dimitrov and H. Haas, Principles of LED Light Communications (Cambridge University Press, 2015).

[15] R. Huang, S. Li, S. Xue, Z. Jiang, and S. Wu, Preparation and characterization of YAG: $\mathrm{Ce} 3+$ phosphors by solsolvothermal process, in: 2012 Int. Conf. Futur. Environ. Energy IPCBEE, (2012), pp. 85-89. 
[16] M. Janulevicius, P. Marmokas, M. Misevicius, J. Grigorjevaite, L. Mikoliunaite, S. Sakirzanovas, and A. Katelnikovas, Sci. Rep. 6(may), 26098 (2016).

[17] C. Weisbuch, M. Piccardo, L. Martinelli, J. Iveland, J. Peretti, and J. S. Speck, Phys. status solidi 212(5), 899913 (2015).

[18] M. Auf der Maur, A. Pecchia, G. Penazzi, W. Rodrigues, and A. Di Carlo, Phys. Rev. Lett. 116(2), 027401 (2016).

[19] OSRAM Opto Semiconductors, Brilliant Mix from OSRAM Opto Semiconductors, 2012, http://ledlight.osram-os. com/technology/brilliant-mix/.

[20] Chih-Feng Lu, Chi-Feng Huang, Yung-Sheng Chen, WenYu Shiao, Cheng-Yen Chen, Yen-Cheng Lu, and ChihChung Yang, IEEE J. Sel. Top. Quantum Electron. 15(4), 1210-1217 (2009).

[21] B. Damilano, P. Demolon, J. Brault, T. Huault, F. Natali, and J. Massies, J. Appl. Phys. 108(7), 073115 (2010).

[22] H.S. El-Ghoroury, C. L. Chuang, and M. V. Kisin, IIInitride monolithic LED covering full RGB color gamut, in: Proc. SPIE-Physics Simul. Optoelectron. Devices XXIV, edited by B. Witzigmann, M. Osiński, and Y. Arakawa (mar 2016), p. 974222

[23] M. L. Lee, Y. H. Yeh, S. J. Tu, P. C. Chen, W. C. Lai, and J. K. Sheu, Opt. Express 23(7), A401 (2015).

[24] S. H. Lim, Y.H. Ko, C. Rodriguez, S. H. Gong, and Y. H. Cho, Light Sci. Appl. 5(2), e16030 (2016).

[25] S. Y. Karpov, N. A. Cherkashin, W. V. Lundin, A. E. Nikolaev, A. V. Sakharov, M. A. Sinitsin, S.O. Usov, E. E. Zavarin, and A. F. Tsatsulnikov, Phys. status solidi 213(1), 19-29 (2016).

[26] H. S. El-Ghoroury, C. L. Chuang, and Z. Y. Alpaslan, Quantum Photonic Imager (QPI): A Novel Display Technology that Enables more than 3D Applications, in: SID Symp. Dig. Tech. Pap., (jun 2015), pp.371-374.

[27] R. Mirhosseini, M. F. Schubert, S. Chhajed, J. Cho, J. K. Kim, and E. F. Schubert, Opt. Express 17(13), 10806 (2009).

[28] P. Stauss, M. Mandl, P. Rode, A. Laubsch, A. Biebersdorf, R. Windisch, B. Galler, P. Drechsel, and U. Steegmüller, Phys. status solidi 8(7-8), 2396-2398 (2011).

[29] C. C. Lin, Y. S. Zheng, H. Y. Chen, C. H. Ruan, G. W. Xiao, and R. S. Liu, J. Electrochem. Soc. 157(9), H900 (2010).

[30] M. Zulonas, T. J. Slight, I. E. Titkov, A. Sakharov, K. A. Fedorova, W. Lundin, A. Nikolaev, W. Meredith, A. Tsatsulnikov, and E. U. Rafailov, Improvement of external quantum efficiency of blue and green InGaN LEDs using corrugated interface substrates, in: Eur. Conf. Lasers ElectroOptics - Eur. Quantum Electron. Conf. (Optical Soc. Am., (2015), p. CE_11_3.

[31] A.F. Tsatsulnikov, W. V. Lundin, A. V. Sakharov, E. E. Zavarin, S. O. Usov, A. E. Nikolaev, N. V. Kryzhanovskaya, V.S. Sizov, M. A. Synitsin, E. V. Yakovlev, A.E. Chernyakov, A.L. Zakgeim, N.A. Cherkashin, and M. Hytch, Phys. status solidi 8(7-8), 2308-2310 (2011).

[32] PhosphorTech Corporation, PhosphorTech Datasheet, 2014.

[33] International Commission on Illumination, Method of measuring and specifying color rendering properties of light sources., Tech. rep.

[34] I. E. Titkov, S. Y. Karpov, A. Yadav, V. L. Zerova, M. Zulonas, B. Galler, M. Strassburg, I. Pietzonka, H. J. Lugauer, and E. U. Rafailov, IEEE J. Quantum Electron. 50(11), 911920 (2014).
[35] A. E. Chernyakov, K. A. Bulashevich, S. Y. Karpov, and A. L. Zakgeim, Phys. status solidi 210(3), 466-469 (2013).

[36] S. Y. Karpov, M. Binder, B. Galler, and D. Schiavon, Phys. status solidi - Rapid Res. Lett. 9(5), 312-316 (2015).

[37] S. Y. Karpov, Light-emitting diodes for solid-state lighting: searching room for improvements, in: Proc. SPIE - Light. Diodes Mater. Devices, Appl. Solid State Light. XX. SPIE San Fr., edited by H. Jeon, L. W. Tu, M. R. Krames, and M. Strassburg (mar 2016), p. 97680C. 